\title{
Intrahepatic Cholestasis of Pregnancy: Advances in Diagnosis and Management
}

\section{Mishra ${ }^{1 *}$ and Rohilla $\mathbf{M}^{2}$}

${ }^{1}$ Former Senior Resident, Department of Obstetrics and Gynaecology, PGIMER, India

${ }^{2}$ Professor, Department of Obstetrics and Gynaecology, PGIMER, India

\section{Mini Review}

Volume 2 Special Issue 3

Received Date: March 15, 2017

Published Date: March 31, 2017

*Corresponding author: Nitu Mishra MS, DNB (Obstetrics and Gynaecology), MNAMS, Former Senior Resident, Department of Obstetrics and Gynaecology, PGIMER, Chandigarh India, 160012, Email: nitumishra07@gmail.com

\section{Abstract}

Intra hepatic cholestasis of pregnancy (ICP) is considered to be the most common liver disease affecting pregnancy. While a number of genetic, hormonal and environmental factors have been found to influence the prevalence of ICP, the aetiology is still not fully known. High levels of circulating estrogen seen during the later part of second trimester and third trimester of pregnancy are believed to play a role in inducing cholestasis. The presenting complaints are of itching especially of palms and soles. Total serum bile acid (TSBA) levels are raised in patients of ICP and TSBA is considered the most sensitive and specific marker for the diagnosis of ICP (fasting serum bile acid concentrations greater than 10 $\mu \mathrm{mol} / \mathrm{L}$ ). While the itching tends to be self limited and often resolves after delivery, it has the tendency of producing adverse effects on the foetus. ICP can lead to spontaneous preterm birth, meconium-stained amniotic fluid, increased rates of caesarean sections and fetal distress which may end up in stillbirth. While a number of therapeutic agents have been used for controlling the itching, only ursodeoxycholic acid (UDCA) has been found to be effective in controlling maternal itching. Termination of pregnancy after completed 37 weeks of gestation is an acceptable line of management. Recent advances have seen attempts to identify the exact etiology of ICP, the best modality for diagnosis and prognostication, and treatment modalities. This article reviews the available diagnostic and management options and looks into the recent advances.

Keywords: Intrahepatic cholestasis of pregnancy; Total serum bile acid; Ursodeoxycholic acid; Still Birth; Fetal Distress

\section{Introduction}

Pregnancy is a unique physiologic condition which involves changes in all the organ systems of the human body. While the body adapts to these changes in most of the times, there are instances when the body cannot and hence, there arise conditions which are seen almost exclusively in pregnancy which tend to improve after child birth. Intra hepatic cholestasis of pregnancy (ICP) is such a condition and is considered to be the most common liver disease affecting pregnancy [1]. 
It has been more than 130 years since the condition was first described, yet the exact aetiology is still to be determined [2].

\section{Epidemiology and Etiopathogenesis}

A number of genetic, hormonal and environmental factors have been found to influence the prevalence of ICP. The prevalence of ICP is highest in South American countries of Chile and Bolivia (1.5-13.2\%), followed by the Scandinavian countries. Prevalence is lowest in US, Southern Europe, Asia and Australia [2,3]. Incidence of ICP is high in women with previous history of intrahepatic cholestasis during earlier pregnancies, with history of cholestasis due to oral contraceptive use, family history of hepatobiliary diseases. Women with in vitro fertilization and with twin pregnancy also have an increased chance of developing ICP $[3,4]$. It has been postulated that high levels of circulating estrogen seen during the later part of second trimester and third trimester of pregnancy plays a role in inducing cholestasis. Genetic mutations in the biliary canalicular transport proteins tend to increase their sensitivity to estrogen and can predispose to ICP [5]. Few extraneous factors have also been suspected in causing the disease. Seasonal variations (with higher prevalences in winter), insufficient dietary selenium intake and increased absorption of bacterial endotoxins and pollutants (such as pesticides) in the gut are few such factors $[2,3]$.

There are lacunae in the current understanding of pathophysiology of ICP. An increased serum bile acid concentration is believed to trigger the onset of itching. However, a correlation between bile acid levels and the severity of itching has not been conclusively demonstrated [4].

\section{Clinical Presentation and Diagnosis}

"Pruritus" or itching classically worse at night and primarily on the palms and soles in the late second trimester and third trimester is the presenting complaint. In very severe cases, itching may affect the ears, the eyelids, and even the oral cavity. Skin rash is usually absent. The patient is rarely jaundiced and other constitutional symptoms of cholestasis are absent too. Liver function tests (LFTs) which estimate the serum bilirubin and transaminase levels are mildly deranged. Thus, the presentation is not significant enough to attract the attention of treating physician lacking in awareness of ICP and exclusion of all other possible causes of pruritus is not always possible. However, total serum bile acid (TSBA) levels are raised in patients of ICP and TSBA is considered the most sensitive and specific marker for the diagnosis of ICP (fasting serum bile acid concentrations greater than $10 \mu \mathrm{mol} / \mathrm{L}$ ) [6]. Also, higher bile acid levels ( $>40 \mu \mathrm{mol} / \mathrm{L}$ ) have been reported to be associated with a higher rate of fetal complications [7].

A well described pathway for diagnosis has been published by the Royal college of Obstetrics and Gynaecology (RCOG) [8]. Based on history, clinical examinations and laboratory investigations attempts are made to pinpoint the diagnosis. Other causes of cholestasis are ruled out by ultrasonography of abdomen and by laboratory tests.

The importance of this pruritic condition of pregnancy is due to the fetal complications associated with it. The maternal outcome is rarely of concern as the itching and deranged LFT has been found to subside spontaneously after delivery. ICP can lead to spontaneous preterm birth, meconium-stained amniotic fluid, increased rates of caesarean sections and fetal distress which may end up in still birth $[2,3,8,9]$. A positive correlation has been found between TSBA levels and fetal complications, with TSBA $>40 \mu \mathrm{mol} / \mathrm{L}$ being associated with significantly higher rates of fetal complications [9]. It has been postulated that a higher level of bile acids in maternal circulation increases the chances of them crossing over to the fetus and hence the increased complications.

\section{Management}

The management of ICP should be considered in two parts. One is the management of itching and second being the attempts to reduce fetal complications. The maternal itching part is addressed by the use of anti-histaminic drugs like hydroxyzine and diphenhydramine, and topical emollients like calamine and paraffin10. Dexamethasone and phenobarbitone has been used aiming to reduce the production of bile acids. Activated charcoal, guar gum, cholestyramine have been used as agents to bind bile acids in the intestine facilitating their elimination [10]. Other agents' ursodeoxycholic acid (UDCA) and Sadenosylmethionine (SAMe) have been postulated to detoxify bile acids, or change their solubility necessary for increased hepatic secretion [10]. Of all these methods of treatment only UDCA has been found to improve the maternal symptom of pruritus in the Cochrane review by Gurung et al. [10]. The recently published meta-analysis by Kong et al. [11] has reported that UDCA is effective and safe to improve pruritus and liver function in ICP and that UDCA also reduces adverse maternal and fetal outcomes in pregnant women with ICP. In patients with prolonged prothrombin time, vitamin $\mathrm{K}$ should be administered. 
A patient with ICP should ideally be managed in an institutional set up with obstetric care expertise. No specific method of antenatal fetal monitoring for the prediction of fetal death has been recommended; ultrasound and cardiotocography have not been found as reliable methods for preventing fetal death by RCOG [8]. As high blood levels of bile acids have been found to be associated with poor fetal outcomes, decision making can be aided by bile acid levels; however, it should not be the sole criteria for decision making. Also, delivery should be contemplated once the fetal maturity has been achieved. An informed decision has to be taken regarding termination of pregnancy. While it is not evidence based, most obstetricians offer termination of pregnancy at completed 37 weeks of gestation [3,8]. A detailed discussion about the pros and cons of termination of pregnancy should be done with the patient with an emphasis on inability to predict the fetal outcome [8].

\section{What is New in ICP??}

A pubmed search with keywords "intrahepatic cholestasis of pregnancy" returned 807 results. Narrowing down the search criteria to look for results of past 5 years returned 315 results; showing that researchers are focussed on solving the mysteries of ICP. The recent research is directed towards identification of a sensitive biomarker of ICP [12], diagnostic modality which could help in prognosis [2], role of UDCA [11,13,14] and attempts to identify factors affecting fetal complications.

A Cochrane database review [2] is in progress with an aim to determine the diagnostic accuracy of total serum bile acids or total serum bile acids profile, or both for the diagnosis of intrahepatic cholestasis of pregnancy in pregnant women presenting with pruritus and to compare the diagnostic accuracy of total serum bile acids and each component of serum bile acid profile in diagnosing intrahepatic cholestasis of pregnancy; to define the optimal cut-off values for these; and to investigate possible sources of heterogeneity. It is expected to resolve many of the diagnostic dilemmas faced by obstetrician's world over.

It is however imperative that patients and healthcare providers are sensitized to ICP and the risks to the fetus brought by it so that any presumed harmless pruritus is not neglected. Such patients should be managed under guidance of an obstetrician. The ongoing research in the field is expected to guide better management of ICP patients.

\section{References}

1. Tran TT, Ahn J, Reau NS (2016) ACG Clinical Guideline: Liver Disease and Pregnancy. Am J Gastroenterol 111(2): 176-194.

2. Manzotti C, Casazza G, Stimac T, Nikolova D, Gluud C (2017) Total serum bile acids or serum bile acid profile, or both, for the diagnosis of intrahepatic cholestasis of pregnancy. Cochrane Database of Systematic Reviews 2: CD012546.

3. Rodrigo Zapata (2015-2017) Intrahepatic cholestasis of Pregnancy: Even Today a Puzzling Disease of Pregnancy. WGO'S.

4. Pathak B, Sheibani L, Lee RH (2010) Cholestasis of pregnancy. Obstetrics and Gynaecology Clinics of North America 37(2): 269-282.

5. Floreani A, Gervasi MT (2016) New Insights on Intrahepatic Cholestasis of Pregnancy. Clin Liver Dis 20(1): 177-189.

6. Diken Z, Usta IM, Nassar AH (2014) A clinical approach to intrahepatic cholestasis of pregnancy. American Journal of Perinatology 31(1): 1-8.

7. Glantz A, Marschall HU, Mattson LA (2004) Intrahepatic cholestasis of pregnancy: relationships observed bile acid levels and fetal complication rates. Hepatology 40(2): 467-474.

8. https://www.rcog.org.uk/globalassets/documents/g uidelines/gtg_43.pdf

9. Glantz A, Marschall HU, Mattson LA (2004) Intrahepatic cholestasis of pregnancy: relationships observed bile acid levels and fetal complication rates. Hepatology 40(2): 467-474.

10. Gurung V, Stokes M, Middleton P, Milan SJ, Hague W, Thornton JG (2013) Interventions for treating cholestasis in pregnancy. Cochrane Database of Systematic Reviews 6: CD000493.

11. Kong X, Kong Y, Zhang F, Wang T, Yan J (2016) Evaluating the effectiveness and safety of ursodeoxycholic acid in treatment of intrahepatic cholestasis of pregnancy: A meta-analysis (a prismacompliant study). Medicine (Baltimore) 95(40): e4949. 
12. Chen Z, Shen Z, Hu L, Lu M, Feng Y (2013) Identification of Matrix Metalloproteinase- 2 and 9 as Biomarker of Intrahepatic Cholestasis of Pregnancy. Ann Hepatol 16(2): 291-296.

13. Bacq Y, le Besco M, Lecuyer AI, Gendrot C, Potin J, et al. (2017) Ursodeoxycholic acid therapy in intrahepatic cholestasis of pregnancy: Results in realworld conditions and factors predictive of response to treatment. Dig Liver Dis 49(1): 63-69.

14. Zhang Y, Lu L, Victor DW, Xin Y, Xuan S (2016) Ursodeoxycholic Acid and S-adenosylmethionine for the Treatment of Intrahepatic Cholestasis of Pregnancy: A Meta-analysis. Hepatitis Monthly 16(8): e38558. 